\title{
NONLINEAR OBSERVERS FOR LOCALLY UNIFORMLY OBSERVABLE SYSTEMS
}

\author{
HASSAN HAMmOURI $^{1}$ AND M. FARZA ${ }^{2}$
}

\begin{abstract}
This paper deals with the observability analysis and the observer synthesis of a class of nonlinear systems. In the single output case, it is known [4-6] that systems which are observable independently of the inputs, admit an observable canonical form. These systems are called uniformly observable systems. Moreover, a high gain observer for these systems can be designed on the basis of this canonical form. In this paper, we extend the above results to multi-output uniformly observable systems. Corresponding canonical forms are presented and sufficient conditions which permit the design of constant and high gain observers for these systems are given.
\end{abstract}

Mathematics Subject Classification. 37N35, 93Bxx.

Received October 21, 2002. Revised December 10, 2002.

\section{INTRODUCTION}

Many techniques have been developed for designing an observer for nonlinear systems. Among these techniques, a rather natural approach consists in considering systems which can be steered by a change of coordinates into state affine systems up to output injection. Indeed, for these systems an extended Luenberger (or Kalman) observer can be designed. Several authors [11,12,14], have characterized such nonlinear systems. An important feature of these systems lies in the fact that they possess similar observability properties as linear ones. Consequently, they are observable independently of the inputs.

Generally, observable nonlinear systems are not diffeomororphic to linear systems up to output injection and may admit singular inputs (i.e. inputs that do not distinguish two initial different states). Up to now, does not exist complete theory which allows the design of an observer for general observable systems. This is partly due to the complexity of the singular inputs analysis. However, for some classes of nonlinear systems, the authors in $[1,2]$ have given sufficient conditions allowing the design of observers which converge irrespectively of the inputs, based on Lyapunov techniques. As it has been noted in [1], these systems are such that if an input $u$ does not distinguish two initial states $x \neq \bar{x}$ on $\mathbb{R}^{+}$, then, their respective trajectories $x^{u}(t), \bar{x}^{u}(t)$, which are issued from these initial states, are such that $\lim _{t \rightarrow+\infty}\left(x^{u}(t)-\bar{x}^{u}(t)\right)=0$. In the linear case, this means that the unobservable modes are stable.

As suggested above, an interesting class of nonlinear systems are those which are observable independently of the inputs. These systems are also called uniformly observable. In the control affine case, the study of such

Keywords and phrases. Nonlinear systems, uniform observability, nonlinear observer.

1 LAGEP, UMR 5007 du CNRS, Université Lyon 1, ESCPE Lyon, bâtiment 308G, 43 boulevard du 11 novembre 1918, 69622 Villeurbanne Cedex, France; e-mail: hammouri@lagep.cpe.fr

2 LAP, EA 2611, ISMRA, Université de Caen, 6 boulevard du Maréchal Juin, 14050 Caen Cedex, France. 
systems started in 1977 when the author in [15] gave a canonical form for single-output uniformly observable bilinear systems. This canonical form was then used to synthesize an observer. Later, the authors in [4] extended this canonical form for single-output uniformly observable nonlinear systems in the control affine case. A new proof of this result is given in [5]. Moreover, using this canonical form, the authors designed a high gain observer. Using similar canonical forms, many authors have studied separately high gain observer synthesis (see for instance $[3,6])$.

Since there is no normal form which characterizes general multi-output uniformly observable systems, one of our objectives, in this paper, consists in the characterization of a large class of uniformly observable systems. Therefore, we introduce the notion of uniform observable structure and give a triangular canonical form which extends the canonical form given in [6].

This paper is organized as follows: in Section 2, we give a brief survey of some basic notions and general results related to the uniform observability and the high gain observer synthesis for some particular class of nonlinear systems. In Section 3, we introduce the canonical form of uniformly observable systems in the multioutput case and we propose two observers synthesis for these systems. The first observer has a constant gain while the second has a gain which depends on the inputs. Appropriate assumptions under which each of these observers can be synthesized are given and discussed. In Section 4, we characterize nonlinear systems that can be steered by a change of coordinates to the considered canonical form.

\section{SOME OBSERVABILITY CONCEPTS AND RELATED RESUltS}

Different notions of observability have been presented in $[9,10]$. More recent concepts of observability can be found in [8]. The purpose of this section is to establish the definitions of some classical and relatively new concepts of observability. We therefore, recall some theoretical results and implications associated to these concepts.

Consider the MIMO nonlinear system:

$$
\left\{\begin{array}{l}
\dot{x}=f(u, x) \\
y=h(x)
\end{array}\right.
$$

$x(t) \in M$, a $n$-dimensional manifold; $u(t) \in U$, a Borelian set of $\mathbb{R}^{m} ; u$ and $y$ are the known input and output of (1) respectively.

Throughout this paper, system (1) is assumed to be smooth. This means that there exists an open set $\tilde{U}$ containing $U$ such that:

are of class $\mathcal{C}^{\infty}$.

$$
f: \tilde{U} \times M \longrightarrow T M \quad \text { and } h: M \longrightarrow \mathbb{R}^{p}
$$

For every fixed $u \in U, f_{u}: M \longrightarrow T M$ denotes the vector field defined by $f_{u}(x)=f(u, x)$ and the map $h=\left(h_{1}, \ldots, h_{p}\right)$ is an almost everywhere local submersion. It means that, $\operatorname{Rank}\left(\frac{\partial h}{\partial x}(x)\right)=p$ for almost every $x$.

\section{- Some well-known observability notions}

Let $u \in L^{\infty}([0, T], U), x \in M$ an initial state and $x^{u}(\cdot)$ the trajectory associated to the initial state $x$ and to the input $u$. This trajectory is well-defined on the maximal interval $[0, T(x, u)[\subset[0, T]$. When $T(u, x)<T$, $T(u, x)$ is called the positive escape time. In such a case, $T(u, x)$ has the following property: for every sequence $\left(t_{n}\right)_{n \geq 0}$ s.t. $\lim _{n \rightarrow+\infty} t_{n}=T(u, x)$, the set $\left\{x^{u}\left(t_{n}\right), n \geq 0\right\}$ has no accumulation point.

System (1) is said to be observable if for every two different initial states $x, \bar{x}$; there exists an input $u \in$ $L^{\infty}([0, T], U)$ s.t. $h\left(x^{u}(\cdot)\right)$ is not identically equal to $h\left(\bar{x}^{u}(\cdot)\right)$ on $[0, T(x, \bar{x}, u)[$ where $T(x, \bar{x}, u)=\min \{T(u, x)$, $T(u, \bar{x})\}$. We say that such an input distinguishes the considered initial states $x, \bar{x}$ on $[0, T]$.

An input which distinguishes every pair of different initial states on $[0, T]$ is called a universal input on $[0, T]$. A non universal input is called a singular input on $[0, T]$. Notice that unlike linear systems, observable nonlinear systems may admit singular inputs. Obviously, a system which admits a universal input is observable. The 
converse is also true in the analytical case (it means $U, M, f$ and $h$ are analytic). The proof of this result is given in [13].

Now, denote by $\mathcal{O}$ the smallest vector space containing $h_{1}, \ldots, h_{p}$ and closed under the Lie derivatives $L_{f_{u}}$, $u \in U$ (i.e. $\forall u \in U ; \forall \tau \in \mathcal{O}, L_{f_{u}}(\tau) \in \mathcal{O}$ ). This vector space is the classical observation space. Let $\tilde{\mathcal{O}}$ be the co-distribution spanned by $\{d \tau, \tau \in \mathcal{O}\}$, system $(1)$ is said to be rank observable at $x \in M$ if $\operatorname{dim} \tilde{\mathcal{O}}(x)=n$. It is said to be rank observable if $\forall x \in M, \operatorname{dim} \tilde{\mathcal{O}}(x)=n$. This rank observability condition is related to the concept of local weak observability notion (see for instance [9] for more details and precise definitions).

\section{- Uniform observability concepts}

The following definitions and results are useful for the characterization of systems of the form (1) which are observable independently on the input.

Definition 2.1. Let $E$ be any borelian subset of $U$, system (1) is said to be:

(i) $E$-uniformly observable iff for every $T>0$ and every $u \in L^{\infty}([0, T], E), u$ is a universal input on $[0, T]$

(ii) locally $E$-uniformly observable iff every $x \in M$ admits an open neighborhood $V_{x}$ s.t. system (1) restricted to $V_{x}$ is $E$-uniformly observable;

(iii) locally $E$-uniformly observable almost everywhere iff there exists an open dense subset $M^{\prime}$ of $M$ s.t. the restriction of system (1) to $M^{\prime}$ is locally $E$-uniformly observable.

For single output control affine systems:

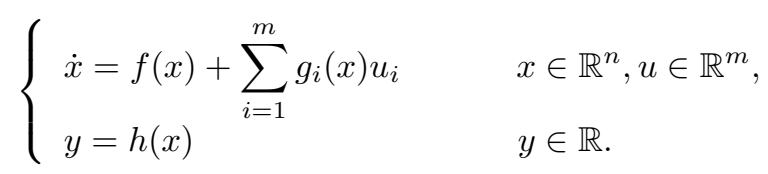

The authors in [4] have characterized systems which are locally $R^{m}$-uniformly observable almost everywhere. They showed that if the system is locally $\mathbb{R}^{m}$-uniformly observable, then locally almost everywhere it can be steered by the local change of coordinates:

$$
z=\left[h(x), L_{f}(h)(x), \ldots, L_{f}^{n-1}(h)(x)\right]^{T}
$$

into:

$$
\left\{\begin{array}{l}
\dot{z}=A z+\varphi(z)+\sum_{i=1}^{m} \psi_{i}(z) u_{i} \\
y=C z
\end{array}\right.
$$

where,

$$
A=\left(\begin{array}{cccc}
0 & 1 & & 0 \\
\vdots & & \ddots & \\
& & & 1 \\
0 & & \ldots & 0
\end{array}\right), \varphi(z)=\left(\begin{array}{c}
0 \\
\vdots \\
\varphi_{n}(z)
\end{array}\right), C=[1,0, \ldots, 0]
$$

and the $j$-th component $\psi_{i j}$ of $\psi_{i}$ is such that $\psi_{i j}(z)=\psi_{i j}\left(z^{1}, \ldots, z^{j}\right)$ for $j=1, \ldots, n ; i=1, \ldots, m$.

A short proof of this result is given in [5]. Moreover, the authors used this triangular canonical form to construct a high gain observer. In the single output case, this canonical form as well as its associated high gain observer have been extended in [6] to single output analytic systems of the form:

$$
\left\{\begin{array}{l}
\dot{x}=f(u, x) \\
y=h(u, x) .
\end{array}\right.
$$


To do so, the authors used the uniform infinitesimal observability concept: consider the tangent map $T f_{u}$ : $T M \longrightarrow T(T M)$ associated to $f_{u}: M \longrightarrow T M$, for every $u \in U$. The family of vector fields $\left(T f_{u}\right)_{u \in U}$, defines, in a unique sense, a lifted system on $T M$ :

$$
\dot{\xi}=T_{M} f_{u}(\xi)
$$

Finally the lifted system associated to system (4) is given by:

$$
\left\{\begin{array}{l}
\dot{\xi}=T_{M} f_{u}(\xi) \\
\tilde{y}=d_{M} h(\xi, u)
\end{array}\right.
$$

where $d_{M} h(\cdot, u)$ is the classical differential map from $T M \longrightarrow \mathbb{R}$.

When $M=\mathbb{R}^{n}, T M$ can be identified with $\mathbb{R}^{n} \times \mathbb{R}^{n}$ and $\xi=(x, z)$. Thus system (5) takes the form:

$$
\left\{\begin{aligned}
\dot{x} & =f(u, x) \\
\dot{z} & =\frac{\partial f}{\partial x}(u, x) \cdot z \\
\tilde{y} & =\frac{\partial h}{\partial x}(u, x) \cdot z
\end{aligned}\right.
$$

Definition $2.2([6])$. Let $u \in L^{\infty}([0, T], U)$ and $x \in M$ :

i) system (4) is said to be infinitesimally observable at $(u, x)$ if the linear map:

$$
T_{x} M \longrightarrow L^{\infty}\left(\left[0, T(u, x)[, \mathbb{R}) \quad\left(\xi \longrightarrow d_{M} h\left(u(\cdot), \xi^{u}(\cdot)\right)\right)\right.\right.
$$

is one to one;

ii) system (4) is called uniformly infinitesimally observable iff for every $T>0$; for every $(u, x) \in$ $L^{\infty}([0, T], U) \times M$, system (4) is infinitesimally observable at $(u, x)$.

The following result is stated in [6] (Th. 3.1):

Theorem 2.1 ( [6]). Assume that the single output system (4) is analytic and uniformly infinitesimally observable and that either one of the following conditions holds:

(i) $U$ is a compact connected analytic manifold;

(ii) $U=\mathbb{R}^{m}$ and $f$, $h$ are polynomial in $u$.

Then, there exists a subanalytic (resp. semi-analytic in the case of (ii)) subset $M^{\prime}$ of codimension 1 in $M$ such that system (4) is locally everywhere diffeomorphic to the triangular canonical form:

$$
\left\{\begin{array}{l}
\dot{z}^{1}=F^{1}\left(u, z^{1}, z^{2}\right) \\
\dot{z}^{2}=F^{2}\left(u, z^{1}, z^{2}, z^{3}\right) \\
\ldots \\
\dot{z}^{i}=F^{i}\left(u, z^{1}, \ldots, z^{i+1}\right) \\
\ldots \\
\dot{z}^{n}=F^{n}\left(u, z^{1}, \ldots, z^{n}\right) \\
y=H\left(u, z^{1}\right)
\end{array}\right.
$$

with

$$
\frac{\partial H}{\partial z^{1}}(u, z) \neq 0 \text { and } \frac{\partial F^{i}}{\partial z^{i+1}}(u, z) \neq 0 ; \forall(u, z) \in U \times V, \text { and } i=1, \ldots, n-1
$$

where $V$ is the domain in which the local transformation takes its values. 
Both canonical forms (3) and (7) have been used to design an observer for single output systems (1). The structure of the observer takes the following form:

$$
\dot{\hat{z}}=F(u, \hat{z})+K(C \hat{z}-y)
$$

where $F(u, z)$ is the dynamics of systems $(3,7)$ and $K$ is a constant vector which does not depend on the input.

In Section 3, we will extend the above observer design (9) to a class of MIMO nonlinear systems which generalizes systems (7). The class of nonlinear systems which can be steered by a change of coordinates to such a canonical form will be characterized in Section 4.

\section{OBSERVER SYNTHESIS}

The canonical form that we consider has the following triangular structure:

$$
\left\{\begin{array}{l}
\dot{z}=F(u, z) \\
y=C z
\end{array}\right.
$$

where $F(u, z)=\left(\begin{array}{c}F^{1}(u, z) \\ \vdots \\ F^{q}(u, z)\end{array}\right), z=\left(\begin{array}{c}z^{1} \\ \vdots \\ z^{q}\end{array}\right) ; u \in U$ a compact submanifold of $\mathbb{R}^{m} ; z^{i} \in \mathbb{R}^{n_{i}} ; n_{1} \geq n_{2} \geq \ldots \geq$ $n_{q} ; n_{1}+\ldots+n_{q}=n$. Each function $F^{i}(u, z), i=1, \ldots, q-1$ satisfies the following structure:

$$
F^{i}(u, z)=F^{i}\left(u, z^{1}, \ldots, z^{i+1}\right), z^{i} \in \mathbb{R}^{n_{i}}
$$

with the following rank condition:

$$
\operatorname{Rank}\left(\frac{\partial F^{i}}{\partial z^{i+1}}(u, z)\right)=n_{i+1} \quad \forall z \in \mathbb{R}^{n} ; \forall u \in U .
$$

In Section 4, we will show that condition (12) characterizes a subclass of locally $U$-uniformly observable systems.

Definition 3.1. A constant gain exponential observer for system (10) is a dynamical system of the form:

$$
\dot{\hat{z}}=F(u, \hat{z})+K(C \hat{z}-y)
$$

where $K$ is a constant matrix such that:

$$
\|\hat{z}(t)-z(t)\| \leq \lambda \mathrm{e}^{-\mu t}\|\hat{z}(0)-z(0)\|
$$

where $\lambda>0$ and $\mu>0$ are constants which do not depend on the input $u \in L_{\infty}\left(\mathbb{R}^{+}, U\right)$ nor on $\hat{z}(0), z(0)$.

In this section, we will give two observer constructions. First, we give a sufficient condition allowing to design a constant gain exponential observer for system (10). Next, we propose an observer construction for general systems of the form (10-12).

\subsection{Constant gain exponential observer}

Consider again system (10) where the inputs $u(t)$ take their values in some Borelian and bounded subset of $\mathbb{R}^{m}$. As in many works related to high gain observer synthesis, we need the following assumption:

H1) Global Lipschitz condition:

$\exists c>0 ; \forall u \in U ; \forall z, z^{\prime} \in \mathbb{R}^{n},\left\|F(u, z)-F\left(u, z^{\prime}\right)\right\| \leq c\left\|z-z^{\prime}\right\|$. 
Notice that such assumption can be omitted in the case where the state of the system lies into a bounded set (this remark is formulated in many papers concerning the high gain observers, see for instance [5]).

Now, let $p_{1} \geq p_{2}$ be two positive integers and denote by $\mathbb{M}\left(p_{1}, p_{2} ; \mathbb{R}\right)$ the space of $p_{1} \times p_{2}$ real matrices. Let $N \in \mathbb{M}\left(p_{1}, p_{2} ; \mathbb{R}\right)$ with $\operatorname{rank}(N)=p_{2}$ and consider the convex cone of $\mathbb{M}\left(p_{1}, p_{2} ; \mathbb{R}\right)$ given by $\mathcal{C}\left(p_{1}, p_{2} ; \alpha ; N\right)=$ $\left\{M \in \mathbb{M}\left(p_{1}, p_{2} ; \mathbb{R}\right) ;\right.$ s.t. $\left.M^{T} N+N^{T} M<\alpha I_{p_{2}}\right\}$ where $\alpha$ is a constant real number and $I_{p_{2}}$ is the $p_{2} \times p_{2}$ identity matrix.

Theorem 3.1. Assume that assumption $H_{1}$ ) holds. Then, a sufficient condition for the existence of a constant gain exponential observer for system (10-12) is:

$$
\left\{\begin{array}{l}
\text { for every } k, 1 \leq k \leq q-1, \text { there exists a } n_{k} \times n_{k+1} \text { constant matrix } S_{k, k+1} \text { such that: } \\
\frac{\partial F^{k}}{\partial z^{k+1}}(u, z) \in \mathcal{C}\left(n_{k}, n_{k+1} ;-1 ; S_{k, k+1}\right) ; \text { for every }(u, z) \in U \times \mathbb{R}^{n}
\end{array}\right.
$$

$n_{1} \geq n_{2} \geq \ldots \geq n_{q}, n_{i}$ is the dimension of $z^{i}$-space.

Remark 3.1. In the single output case, condition (8) is equivalent to condition (14) of Theorem 3.1.

The proof of the theorem requires the following proposition:

Proposition 3.1. Assume that $\boldsymbol{H 1}$ ) and (14) hold. Then, there exists a $n \times n$ S.P.D. matrix $P$ satisfying the following condition:

There exist $\rho>0 ; \eta>0$ such that for every $(u, z) \in U \times \mathbb{R}^{n}$, we have:

$$
P A(u, z)+A^{T}(u, z) P-\rho C^{T} C \leq-\eta I
$$

where

$$
A(u, z)=\left(\begin{array}{cccccc}
0 & A_{1}(u, z) & 0 & \ldots & & 0 \\
\vdots & 0 & A_{2}(u, z) & 0 & \ldots & \vdots \\
\vdots & \ldots & \ddots & \ddots & \ddots & \vdots \\
\vdots & \ldots & \ldots & \ddots & \ddots & 0 \\
\vdots & \ldots & \ldots & \ddots & \ddots & A_{q-1}(u, z) \\
0 & \ldots & \ldots & \ldots & \ldots & 0
\end{array}\right)
$$

with $A_{k}(u, z)=\frac{\partial F^{k}}{\partial z^{k+1}}(u, z)$.

Proof of Proposition 3.1. Set $\Gamma_{k}=\left\{\frac{\partial F^{k}}{\partial z^{k+1}}(u, z) ;(u, z) \in U \times \mathbb{R}^{n}\right\}$. From condition (14), we can choose matrices $S_{k, k+1}, 1 \leq k \leq q-1$, such that:

$$
\forall M_{k} \in \Gamma_{k}, S_{k, k+1}^{T} M_{k}+M_{k}^{T} S_{k, k+1}<-I_{n_{k+1}} .
$$


Now consider the following symmetric bloc tridiagonal matrix:

$$
P=\left(\begin{array}{cccccc}
P_{11} & P_{12} & 0 & \ldots & \ldots & 0 \\
P_{12}^{T} & P_{22} & P_{23} & 0 & \ldots & 0 \\
0 & \ddots & \ddots & \ddots & \ddots & \vdots \\
\vdots & \ddots & \ddots & \ddots & \ddots & 0 \\
0 & \ldots & 0 & P_{q-2, q-1}^{T} & P_{q-1, q-1} & P_{q-1, q} \\
0 & \ldots & 0 & 0 & P_{q-1, q}^{T} & P_{q, q}
\end{array}\right)
$$

where $P_{k, k+1}=\rho_{k+1} S_{k, k+1}$ and $P_{k k}$ is a $n_{k} \times n_{k}$ S.D.P. matrix, $\rho_{k+1}$ and $P_{k k}$ will be specified below.

In the sequel, if $M$ is a $k \times l$ matrix, we denote by $\|M\|$ the subordinate \|\|$_{2}$-norm: $\|M\|=\sup _{\|\xi\|=1}\|M \xi\|$, where $\|\xi\|$ and $\|M \xi\|$ are the $L_{2}$-norms.

Let $\sigma_{k}=\lambda_{\max }\left(P_{k k}\right)$ and $\widehat{\sigma_{k}}=\lambda_{\min }\left(P_{k k}\right)$ be the respective largest and smallest eigenvalues of $P_{k k}$. From hypothesis H1), we know that $\Gamma_{k}$ is a bounded subset of $M\left(n_{k}, n_{k+1}, \mathbb{R}\right)$. Set $m_{k}=\sup \left\{\left\|M_{k}\right\| ; M_{k} \in \Gamma_{k}\right\}$ and choose $P$ such that:

(i) $4 \rho_{k+1}^{2}\left\|S_{k, k+1}\right\|^{2}<\widehat{\sigma}_{k} \widehat{\sigma}_{k+1}$, for $1 \leq k \leq q-1$;

(ii) $4 \sigma_{k}^{2} m_{k}^{2}<\rho_{k} \rho_{k+1}$, for $1 \leq k \leq q-1$;

(iii) $4 \rho_{k+1}^{2} m_{k+1}^{2}\left\|S_{k, k+1}\right\|^{2}<\rho_{k} \rho_{k+2}$, for $1 \leq k \leq q-2$.

To obtain such a matrix, it suffices to choose $P_{k k}=\sigma_{k} I_{k}\left(\sigma_{k}=\widehat{\sigma}_{k}\right)$, and numbers $\rho_{k}$ such that $\rho_{k} \ll \sigma_{k} \ll$ $\rho_{k+1} \ll \sigma_{k+1}$, where the notation $a \ll b$ means that $\frac{a}{b}$ is sufficiently small.

Before proving inequality (15) of Proposition 3.1, let us show that $P$ is a S.D.P. matrix. Indeed, let $x \in \mathbb{R}^{n}$, $x \neq 0$, a simple calculation shows that:

$$
\begin{aligned}
x^{T} P x & =\sum_{1}^{q}\left(x^{k^{T}} P_{k k} x^{k}\right)+2 \sum_{1}^{q-1}\left(x^{k^{T}} P_{k, k+1} x^{k+1}\right) \\
& =\frac{1}{2} x^{1^{T}} P_{11} x^{1}+\frac{1}{2} x^{q T} P_{q q} x^{q}+\frac{1}{2} \sum_{1}^{q-1}\left(x^{k^{T}} P_{k k} x^{k}+4 x^{k^{T}} P_{k, k+1}^{T} x^{k+1}+x^{k+1^{T}} P_{k+1, k+1} x^{k+1}\right) \\
& \geq \frac{\widehat{\sigma}_{1}}{2}\left\|x^{1}\right\|^{2}+\frac{\widehat{\sigma}_{q}}{2}\left\|x^{q}\right\|^{2}+\frac{1}{2} \sum_{1}^{q-1}\left(\widehat{\sigma}_{k}\left\|x^{k}\right\|^{2}-4 \rho_{k+1}\left\|S_{k, k+1}\right\|\left\|x^{k}\right\|\left\|x^{k+1}\right\|+\widehat{\sigma}_{k+1}\left\|x^{k+1}\right\|^{2}\right)
\end{aligned}
$$

Using condition (i) above, we get $x^{T} P x>0$.

Now let us show inequality (15) of Proposition 3.1. 
Set $A(u, z)=A$, a simple computation gives:

$$
\begin{aligned}
& x^{T}\left(P A+A^{T} P-\rho_{1} C^{T} C\right) x=-\rho_{1}\left\|x^{1}\right\|^{2}+2 x^{1^{T}} P_{11} A_{1} x^{2}+2 x^{1^{T}} P_{12} A_{2} x^{3} \\
& +x^{2^{T}}\left(P_{12}^{T} A_{1}+A_{1}^{T} P_{12}\right) x^{2}+2 x^{2^{T}} P_{22} A_{2} x^{3}+2 x^{2^{T}} P_{23} A_{3} x^{4} \\
& +\ldots \\
& +x^{q-2^{T}}\left(P_{q-3, q-2}^{T} A_{q-3}+A_{q-3}^{T} P_{q-3, q-2}\right) x^{q-2} \\
& +2 x^{q-2^{T}} P_{q-2, q-2} A_{q-2} x^{q-1}+2 x^{q-2^{T}} P_{q-2, q-1} A_{q-1} x^{q} \\
& +x^{q-1^{T}}\left(P_{q-2, q-1}^{T} A_{q-2}+A_{q-2}^{T} P_{q-2, q-1}\right) x^{q-1} \\
& +2 x^{q-1}{ }^{T} P_{q-1, q-1} A_{q-1} x^{q}+x^{q^{T}}\left(P_{q-1, q}^{T} A_{q-1}+A_{q-1}^{T} P_{q-1, q}\right) x^{q} \\
& =\frac{1}{2}\left\{-\rho_{1}\left\|x^{1}\right\|^{2}+4 x^{1^{T}} P_{11} A_{1} x^{2}+x^{2^{T}}\left(P_{12}^{T} A_{1}+A_{1}^{T} P_{12}\right) x^{2}\right\} \\
& +\frac{1}{2}\left\{-\rho_{1}\left\|x^{1}\right\|^{2}+4 x^{1^{T}} P_{12} A_{2} x^{3}+x^{3^{T}}\left(P_{23^{T}} A_{2}+A_{2}^{T} P_{23}\right) x^{3}\right\} \\
& +\frac{1}{2}\left\{x^{2^{T}}\left(P_{12}^{T} A_{1}+A_{1}^{T} P_{12}\right) x^{2}+4 x^{2^{T}} P_{22} A_{2} x^{3}+x^{3^{T}}\left(P_{23}^{T} A_{2}+A_{2}^{T} P_{23}\right) x^{3}\right\} \\
& +\ldots \\
& +\frac{1}{2}\left\{x^{q-2^{T}}\left(P_{q-3, q-2}^{T} A_{q-3}+A_{q-3}^{T} P_{q-3, q-2}\right) x^{q-2}+4 x^{q-2^{T}} P_{q-2, q-2} A_{q-2} x^{q-1}\right. \\
& \left.+x^{q-1^{T}}\left(P_{q-2, q-1}^{T} A_{q-2}+A_{q-2}^{T} P_{q-2, q-1}\right) x^{q-1}\right\} \\
& +\frac{1}{2}\left\{x^{q-2^{T}}\left(P_{q-3, q-2}^{T} A_{q-3}+A_{q-3}^{T} P_{q-3, q-2}\right) x^{q-2}\right. \\
& \left.+4 x^{q-2^{T}} P_{q-2, q-1} A_{q-1} x^{q}+x^{q T}\left(P_{q-1, q}^{T} A_{q-1}+A_{q-1}^{T} P_{q-1, q}\right) x^{q}\right\} \\
& +\frac{1}{2}\left\{x^{q-1 T}\left(P_{q-2, q-1}^{T} A_{q-2}+A_{q-2}^{T} P_{q-2, q-1}\right) x^{q-1}\right. \\
& \left.+4 x^{q-1^{T}} P_{q-1, q-1} A_{q-1} x^{q}+x^{q T}\left(P_{q-1, q}^{T} A_{q-1}+A_{q-1}^{T} P_{q-1, q}\right) x^{q}\right\} \\
& \leq \frac{1}{2} \sum_{1}^{q-1}\left\{-\rho_{k}\left\|x^{k}\right\|^{2}+4\left\|x^{k}\right\|\left\|x^{k+1}\right\| \sigma_{k} m_{k}-\rho_{k+1}\left\|x^{k+1}\right\|^{2}\right\} \\
& +\frac{1}{2} \sum_{1}^{q-2}\left\{-\rho_{k}\left\|x^{k}\right\|^{2}+4\left\|x^{k}\right\|\left\|x^{k+2}\right\| \rho_{k+1} m_{k+1}\left\|S_{k, k+1}\right\|-\rho_{k+2}\left\|x^{k+2}\right\|^{2}\right\} .
\end{aligned}
$$

Using conditions (ii) and (iii) above, we obtain: $x^{T}\left(P A+A^{T} P-\rho_{1} C^{T} C\right) x \leq-\eta I$, where $\eta>0$ is a constant. This ends the proof of the proposition.

Proof of Theorem 3.1. We assume that hypothesis H1) and condition (14) of Theorem 3.1 hold, and we will construct a constant matrix $K$, such that the following system:

$$
\dot{\hat{z}}=F(u, \hat{z})-\Delta_{\theta} K(C \hat{z}-y)
$$


is a constant gain exponential observer, where $\Delta_{\theta}=\left(\begin{array}{cccc}\theta I_{n_{1}} & 0 & \ldots & 0 \\ 0 & \theta^{2} I_{n_{2}} & 0 & \vdots \\ \vdots & & \ddots & 0 \\ 0 & \ldots & 0 & \theta^{q} I_{n_{q}}\end{array}\right), I_{n_{k}}$ is the $n_{k} \times n_{k}$ identity matrix, $k=1, \ldots, q$ and $\theta>0$ is a constant real number.

Let $P$ be the S.D.P. matrix given by (16) and set $K=P^{-1} C^{T}$. We will show that for $\theta$ sufficiently large, $\hat{z}(t)-z(t)$ exponentially converges to 0 .

As in [5-7] and many other references related to high gain observer synthesis, consider the change of coordinates $\hat{z}=\Delta_{\theta}^{-1} \hat{z}, \bar{z}=\Delta_{\theta}^{-1} z$, and set $\varepsilon=\hat{z}-\bar{z}$. Let us show that $\varepsilon(t)$ exponentially converges to 0 , for $\theta$ sufficiently large.

Set $\delta F=\left(\begin{array}{l}\delta F^{1} \\ \vdots \\ \delta F^{q}\end{array}\right)$, where $\delta F^{i}=F^{i}\left(u, \hat{z}^{1}, \ldots, \hat{z}^{i-1}, z^{i+1}\right)-F^{i}\left(u, z^{1}, \ldots, z^{i-1}, z^{i+1}\right)$ for $1 \leq i \leq q-1$ and $\delta F^{q}=F^{q}(u, \hat{z})-F^{q}(u, z)$.

A simple calculation gives:

$$
\dot{\varepsilon}(t)=\theta\left(A(t)-\rho P^{-1} C^{T} C\right) \varepsilon+\Delta_{\theta}^{-1} \delta F
$$

where

$$
A(t)=\left(\begin{array}{llllll}
0 & \frac{\partial F^{1}}{\partial z^{2}}\left(u, \hat{z}^{1}, \xi^{2}\right) & 0 & \ldots & & 0 \\
\vdots & 0 & \frac{\partial F^{2}}{\partial z^{3}}\left(u, \hat{z}^{1}, \hat{z}^{2}, \xi^{3}\right) & 0 & \ldots & \vdots \\
\vdots & \ldots & \ddots & \ddots & \ddots & \vdots \\
\vdots & \ldots & \ldots & \ddots & & 0 \\
\vdots & \ldots & \ldots & \ddots & & \frac{\partial F^{q-1}}{\partial z^{q}}\left(u, \hat{z}^{1}, \ldots, \hat{z}^{q-1}, \xi^{q}\right) \\
0 & \ldots & \ldots & \ldots & \ldots & 0
\end{array}\right)
$$

with $\xi^{i}=\hat{z}^{i+1}+\omega_{i}\left(\hat{z}^{i+1}-z^{i+1}\right)$ and $\omega_{i}$ is a diagonal matrix whose elements are in $[0,1]$.

To show the exponential convergence to zero of $\varepsilon(t)$, it suffices to show:

$$
\frac{\mathrm{d}}{\mathrm{d} t}\left(\varepsilon^{T}(t) P \varepsilon(t)\right) \leq-\alpha \varepsilon^{T}(t) P \varepsilon(t)
$$

for some constant $\alpha>0$.

Set $V(t)=\varepsilon^{T}(t) P \varepsilon(t)$, we obtain:

$$
\dot{V}(t)=-\theta \varepsilon^{T}(t)\left(A^{T}(t) P-P A(t)-\rho C^{T} C\right) \varepsilon(t)+2 \varepsilon(t) P \Delta_{\theta}^{-1} \delta F .
$$

Using the Lipschitz condition (assumption H1)) and the triangular structure (11), it is not difficult to see that there exists a constant $\beta>0$, such that for every $\theta \geq 1$, we have:

$$
\left\|\Delta_{\theta}^{-1} \delta F\right\| \leq \beta\|\varepsilon\|
$$

where $\beta$ is a constant which only depends on the Lipschitz constant of $F$. 
Combining $(15,20)$ and $(21)$, we deduce:

$$
\dot{V}(t) \leq(-\theta \eta+2 \beta\|P\|)\|\varepsilon(t)\|^{2} \leq \frac{1}{\lambda_{\min }(P)}(-\theta \eta+2 \beta\|P\|) V(t) .
$$

To end the proof of the theorem, it suffices to take $\theta>\max \left(1,2 \frac{\beta}{\eta}\|P\|\right)$.

In this subsection, we have shown that the design of a constant high gain observer requires condition (14). However, this condition is not always satisfied by general systems of the form (10-12).

Indeed, consider the following system:

$$
\left\{\begin{array}{l}
\dot{z^{1}}=u_{1} z^{3} \\
\dot{z^{2}}=u_{2} z^{3} \\
\dot{z^{3}}=0 \\
y=C z=\left(\begin{array}{l}
z^{1} \\
z^{2}
\end{array}\right)
\end{array}\right.
$$

where $u=\left(u_{1}, u_{2}\right)$ belongs to the unit circle $U=\{u$ s.t. $\|u\|=1\}$.

It is obvious to see that system (23) is of the form (10-12) and satisfies hypothesis H1).

Now, assume that system (23) admits a constant gain exponential observer:

$$
\dot{\hat{z}}=A(u) \hat{z}+K(C \hat{z}-y)
$$

where, $A(u)=\left(\begin{array}{ccc}0 & 0 & u_{1} \\ 0 & 0 & u_{2} \\ 0 & 0 & 0\end{array}\right), K=\left(\begin{array}{cc}k_{11} & k_{12} \\ k_{21} & k_{22} \\ k_{31} & k_{32}\end{array}\right)$ is a constant matrix and $C=\left(\begin{array}{ccc}1 & 0 & 0 \\ 0 & 1 & 0\end{array}\right)$.

Thus, for every $u \in L^{\infty}\left(\mathcal{R}^{+}, U\right)$, the error equation:

$$
\dot{e}=(A(u)+K C) e
$$

is exponentially stable at the origin.

In particular, the error equations associated to inputs $u(t)=(1,0)$ and $u(t)=(-1,0)$ are exponentially stable. This implies that:

$$
\left(\begin{array}{lll}
k_{11} & k_{12} & 1 \\
k_{21} & k_{22} & 0 \\
k_{31} & k_{32} & 0
\end{array}\right) \text { and }\left(\begin{array}{ccc}
k_{11} & k_{12} & -1 \\
k_{21} & k_{22} & 0 \\
k_{31} & k_{32} & 0
\end{array}\right) \text { are both Hurwitz matrices. }
$$

A simple calculation shows that this yields to the following contradiction: $k_{21} k_{32}-k_{31} k_{22}<0$ and $k_{21} k_{32}-$ $k_{31} k_{22}>0$.

The next section gives a method allowing to design an exponential observer for systems of the form (10-12).

\subsection{Observer design for systems of the form (10-12)}

Consider again systems of the form (10-12). We know that for every $\left(u, z^{1}, \ldots, z^{k}\right)$ and for $1 \leq k \leq q-1$, the functions $z^{k+1} \mapsto F^{k}\left(u, z^{1}, \ldots,, z^{k}, z^{k+1}\right)$ are locally one to one. In the sequel, we will assume the following:

H2) For $1 \leq k \leq q-1, F^{k}\left(u, z^{1}, \ldots,, z^{k}\right.$, .) is one to one from $\mathbb{R}^{n_{k+1}}$ into $\mathbb{R}^{n_{k}}$.

Notice that in the single output case, condition (i) (resp. (ii)) with condition (8) of Theorem 2.1 imply H2).

Before giving our candidate observer, we need some notations and assumptions. 
Consider the following functions:

$$
\begin{aligned}
& \Phi^{1}\left(u, z^{1}\right)=z^{1} \\
& \Phi^{k}\left(u, z^{1}, \ldots, z^{k}\right)=\frac{\partial \Phi^{k-1}}{\partial z^{k-1}}\left(u, z^{1}, \ldots, z^{k-1}\right) F^{k-1}\left(u, z^{1}, \ldots, z^{k}\right) ; 2 \leq k \leq q
\end{aligned}
$$

From the triangular structure (11), it is easy to see that

$$
\Phi^{k}\left(u, z^{1}, \ldots, z^{k}\right)=\frac{\partial F^{1}}{\partial z^{2}}\left(u, z^{1}, z^{2}\right) \ldots \frac{\partial F^{k-2}}{\partial z^{k-1}}\left(u, z^{1}, \ldots, z^{k-1}\right) F^{k-1}\left(u, z^{1}, \ldots, z^{k}\right)
$$

Using assumption H2) and the rank condition (12), it easy to show that:

- for every $\left(u, z^{1}, \ldots, z^{k-1}\right), \Phi^{k}\left(u, z^{1}, \ldots, z^{k-1},.\right)$ is one to one from $\mathbb{R}^{n_{k}}$ into $\mathbb{R}^{n_{k-1}}$;

- for every $\left(u, z^{1}, \ldots, z^{k-1}\right), \zeta^{k}=\Phi^{k}\left(u, z^{1}, \ldots, z^{k-1}, z^{k}\right)$ implies that $z^{k}=\varphi^{k}\left(u, z^{1}, \ldots, z^{k-1}, \zeta^{k}\right)$ where $\varphi^{k}\left(u, z^{1}, \ldots, z^{k-1}, \zeta^{k}\right)$ is a function which smoothly depends on $\left(u, z^{1}, \ldots, z^{k-1}\right)$.

In the sequel, we will assume that $\varphi^{k}$ admits a smooth extension $\widetilde{\varphi}^{k}$ i.e.

$-\widetilde{\varphi}^{k}$ is a smooth function w.r.t. $\left(u, \zeta^{1}, \ldots, \zeta^{k-1}, \zeta^{k}\right)$;

- moreover, if $\zeta^{i}=\Phi^{i}\left(u, z^{1}, \ldots, z^{i}\right)$ for $1 \leq i \leq k$, then $z^{k}=\widetilde{\varphi}^{k}\left(u, z^{1}, \ldots, z^{k-1}, \zeta^{k}\right)$.

Our candidate observer for system (10-12) takes the following form:

$$
\dot{\hat{z}}=F(u, \hat{z})-\Lambda(u, \hat{z}) \Delta_{\theta} \widetilde{K}(C \hat{z}-y)
$$

where $F$ is given in $(10) ; \Lambda(u, \hat{z})=\left[\left(\frac{\partial \Phi}{\partial z}(u, \hat{z})\right)^{T} \frac{\partial \Phi}{\partial z}(u, \hat{z})\right]^{-1}\left(\frac{\partial \Phi}{\partial z}(u, \hat{z})\right)^{T}$ with

$$
\Phi=\left(\begin{array}{c}
\Phi^{1} \\
\Phi^{2} \\
\vdots \\
\Phi^{q}
\end{array}\right), \Delta_{\theta}=\left(\begin{array}{cccc}
\theta I_{n_{1}} & 0 & \cdots & 0 \\
0 & \theta^{2} I_{n_{1}} & 0 & \vdots \\
\vdots & & \ddots & 0 \\
0 & \ldots & 0 & \theta^{q} I_{n_{1}}
\end{array}\right), I_{n_{1}} \text { is the } n_{1} \times n_{1} \text { identity matrix. }
$$

$\widetilde{K}$ is a $q n_{1} \times n_{1}$ constant matrix such that $\widetilde{A}-\widetilde{K} \widetilde{C}$ is Hurwitz, where $\widetilde{A}$ and $\widetilde{C}$ are respectively $q n_{1} \times q n_{1}$ and $n_{1} \times q n_{1}$ matrices defined by:

$$
\begin{gathered}
\widetilde{A}=\left(\begin{array}{cccccc}
0 & I_{n_{1}} & 0 & \ldots & & 0 \\
\vdots & 0 & I_{n_{1}} & 0 & \ldots & \vdots \\
\vdots & \ldots & \ddots & \ddots & \ddots & \vdots \\
\vdots & \ldots & \ldots & \ddots & \ddots & 0 \\
\vdots & \ldots & \ldots & \ldots & \ddots & I_{n_{1}} \\
0 & \ldots & \ldots & \ldots & \ldots & 0
\end{array}\right) \\
\widetilde{C}=\left(\begin{array}{llll}
I_{n_{1}} & 0 & \ldots & 0
\end{array}\right) .
\end{gathered}
$$

In order to prove the convergence of the above observer, we need some notations and assumptions. 
Consider the following functions defined on $U \times \mathbb{R}^{m} \times \mathbb{R}^{n}$ :

$$
\begin{aligned}
G^{1}(u, v, \zeta)= & G^{1}\left(\zeta^{1}\right)=0 \\
G^{k}(u, v, \zeta)= & G^{k}\left(u, v, \zeta^{1}, \ldots, \zeta^{k}\right)=\frac{\partial \Phi^{k}}{\partial u}\left(u, \zeta^{1}, \widetilde{\varphi}^{2}\left(u, \zeta^{1}, \zeta^{2}\right), \ldots, \widetilde{\varphi}^{k}\left(u, \zeta^{1}, \ldots, \zeta^{k}\right)\right) v \\
& +\sum_{1}^{k-1} \frac{\partial \Phi^{k}}{\partial z^{i}}\left(u, \zeta^{1}, \widetilde{\varphi}^{2}\left(u, \zeta^{1}, \zeta^{2}\right), \ldots, \widetilde{\varphi}^{k}\left(u, \zeta^{1}, \ldots, \zeta^{k}\right)\right) \zeta^{i+1} ; 2 \leq k \leq q .
\end{aligned}
$$

As in the previous section, let us assume the following:

H3) (i) $\exists \alpha>0 ; \forall u \in U ; \forall z, z^{\prime},\left\|\Phi^{k}\left(u, z^{1}, \ldots, z^{k}\right)-\Phi^{k}\left(u, z^{\prime 1}, \ldots, z^{\prime k}\right)\right\| \geq \alpha\left\|z-z^{\prime}\right\|$, for $1 \leq k \leq q$;

(ii) $\forall \rho>0 ; \exists \beta>0 ; \forall u \in U ; \forall v \in \mathbb{R}^{m},\|v\| \leq \rho ; \forall \zeta, \zeta^{\prime} ;\left\|G^{k}(u, v, \zeta)-G^{k}\left(u, v, \zeta^{\prime}\right)\right\| \leq \beta\left\|\zeta-\zeta^{\prime}\right\|$, for $1 \leq k \leq q$.

Notice that condition (i) of (H3) implies that for every $u \in U$, the embedding map $z \mapsto \Phi(u, z)$ preserves the uniform topology.

In the sequel, we denote by $\mathcal{U}$ the set of bounded absolutely continuous functions $u($.$) from \mathbb{R}^{+}$into $U$, with bounded derivatives (i.e. $\dot{u} \in L^{+\infty}\left(\mathbb{R}^{+}\right)$).

Now, we can state our main results:

Theorem 3.2. Assume that system (10-12) satisfies hypotheses $\boldsymbol{H 2}$ ) and $\boldsymbol{H} 3)$, then: for every $u \in \mathcal{U}, \exists \theta_{0}>0$; $\forall \theta>\theta_{0} ; \exists \lambda>0, \exists \sigma>0$,

$$
\|z(t)-\hat{z}(t)\| \leq \lambda \mathrm{e}^{-\sigma t}\|z(0)-\hat{z}(0)\|, \text { for every } t \geq 0 .
$$

Moreover, $\sigma$ may be chosen large by taking $\theta$ sufficiently large.

If we omit hypothesis H3), then we can state:

Corollary 3.1. Consider system (10-12), and assume that H2) is satisfied. Let $u \in \mathcal{U}$ such that every trajectory associated to $u$ and issued from a given compact subset $K_{1}$, lies into a compact subset $K_{2}$. Then, an exponential observer of the form (27) can be designed in order to estimate such bounded trajectories.

Proof of Theorem 3.2. Let $u \in \mathcal{U}$ and consider the following systems:

$$
\begin{aligned}
& \left\{\begin{aligned}
\dot{\zeta} & =\widetilde{A} \zeta+G(u, \dot{u}, \zeta) \\
y & =\widetilde{C} \zeta
\end{aligned}\right. \\
& \dot{\hat{\zeta}}=\widetilde{A} \hat{\zeta}+G(u, \dot{u}, \hat{\zeta})-\Delta_{\theta} \widetilde{K}(\widetilde{C} \hat{\zeta}-y)
\end{aligned}
$$

where, $G=\left(\begin{array}{c}G^{1} \\ G^{2} \\ \vdots \\ G^{q}\end{array}\right)$; the $G^{k}$,s are defined in (30) and $\widetilde{A}, \widetilde{C}$ are given by (28) and (29), and $\widetilde{K}$ is such that $\widetilde{A}-\widetilde{K} \widetilde{C}$ is Hurwitz.

We can easily check that that if $z(t)$ (resp. $\hat{z}(t)$ ) is a trajectory of system (10) (resp. of system (27)) associated to an input $u \in \mathcal{U}$, then $\Phi(u(t), z(t)$ ) (resp. $\Phi(u(t), \hat{z}(t))$ ) is also a trajectory of system (31) (resp. of system (32)). According to hypothesis H3-(i), if $\|\hat{\zeta}(t)-\zeta(t)\|$ exponentially converges to zero, then so does $\|\hat{z}(t)-z(t)\|$. Hence, it suffices to show that system (32) is an exponential observer for (31). 
To do so, we shall proceed as in [5] and [6]. Set $\varepsilon(t)=\Delta_{\theta}^{-1}(\hat{\zeta}(t)-\zeta(t))$, we obtain:

$$
\dot{\varepsilon}=\theta(\widetilde{A}-\widetilde{K} \widetilde{C}) \varepsilon+\Delta_{\theta}^{-1} \delta G
$$

where $\delta G=G(u, \dot{u}, \hat{\zeta})-G(u, \dot{u}, \zeta)$

Since $\widetilde{A}-\widetilde{K} \widetilde{C}$ is Hurwitz, there exists a S.P.D. matrix $P$ such that $P(\widetilde{A}-\widetilde{K} \widetilde{C})+(\widetilde{A}-\widetilde{K} \widetilde{C})^{T} P=-I$ where $I$ is the identity matrix. To end the proof of the exponential convergence, it suffices to show the following:

$$
\frac{\mathrm{d}\left(\varepsilon^{T} P \varepsilon\right)}{\mathrm{d} t}(t) \leq-\mu\|\varepsilon(t)\|^{2}
$$

for $\theta$ sufficiently large and for some constant $\mu>0$.

A simple calculation yields to:

$$
\frac{\mathrm{d}\left(\varepsilon^{T} P \varepsilon\right)}{\mathrm{d} t}(t)=-\theta\|\varepsilon\|^{2}+2 \varepsilon^{T} P \Delta_{\theta}^{-1} \delta G .
$$

As in the proof of Theorem 3.1, using the triangular structure of $G(u, \dot{u}, \zeta)$ w.r.t. $\zeta$ and hypothesis H3)-(ii), and taking $\theta \geq 1$, it follows that

$$
\left\|\Delta_{\theta}^{-1} \delta G\right\| \leq \tilde{\beta}\|\varepsilon\|
$$

where $\tilde{\beta}$ is a constant which does not depend on $\theta$. Combining (35) and (36), we obtain:

$$
\frac{\mathrm{d}\left(\varepsilon^{T} P \varepsilon\right)}{\mathrm{d} t}(t) \leq(-\theta+2 \tilde{\beta})\|\varepsilon\|^{2} .
$$

Now, let us choose $\theta_{0}>\max \{2 \tilde{\beta}, 1\}$, it follows that for $\theta>\theta_{0}$, we have:

$$
\|\varepsilon(t)\| \leq \lambda_{1} \mathrm{e}^{-\lambda_{2} t}\|\varepsilon(0)\|
$$

where $\lambda_{1}>0, \lambda_{2}>0$ are constants, and $\lambda_{2}=\lambda_{2}(\theta) \rightarrow+\infty$ as $\theta \rightarrow+\infty$.

Proof of Corollary 3.1. Let $u \in \mathcal{U}$ and consider the functions $\Phi^{k}$ defined in (26). Let $\Omega_{k}$ be a compact set containing all $\left\{\left(\Phi^{1}(u(t), z(t)), \ldots, \Phi^{k}(u(t), z(t))\right)\right\}$, where $z(t)$ is any trajectory of system (10), associated to the input $u$ and issued from $K_{1}$. Let $\Xi_{k}$ be any $\mathcal{C}^{1}$-function which takes value 1 on $\Omega_{k}$ and vanishes outside a bounded open set containing $\Omega_{k}$. By construction of the system of coordinates $\left(\zeta^{1}, \ldots, \zeta^{q}\right)$ (see above), $\Xi^{k}$ is a function only of $\left(\zeta^{1}, \ldots, \zeta^{k}\right)$ and having a compact support.

$$
\text { Now set } \left.\widetilde{G}(u, \dot{u}, \zeta)=\left(\begin{array}{c}
\Xi^{1} \cdot G^{1}(u, \dot{u}, \zeta) \\
\vdots \\
\Xi^{q} \cdot G^{q}(u, \dot{u}, \zeta)
\end{array}\right) \text { (the } G^{k}(u, \dot{u}, \zeta) \text { 's are given by }(30)\right) \text {, then for every trajectory } z(t)
$$

of system (10) associated to $u$ and issued from $K_{1}, \Phi(u(t)(t), z(t))$ is also a trajectory of the following system:

$$
\left\{\begin{array}{l}
\dot{\zeta}=\widetilde{A} \zeta+\widetilde{G}(u, \dot{u}, \zeta) \\
y=\widetilde{C} \zeta
\end{array}\right.
$$

Thus, it suffices to construct an exponential observer for system (38). 
Now since $\widetilde{G}$ has a triangular structure similar to that of $G$ and since it is a global Lipschitz function w.r.t. $\zeta$, we can proceed in a similar way as above to show that the following system:

$$
\dot{\hat{\zeta}}=\widetilde{A} \hat{\zeta}+\widetilde{G}(u, \dot{u}, \hat{\zeta})-\Delta_{\theta}^{-1} \widetilde{K}(\widetilde{C} \zeta-y)
$$

forms an exponential observer for system (38).

\section{UNIFORM OBSERVABILITY STRUCTURE}

Many observability concepts are stated in Section 2. In this section, we will characterize systems (1) which can be steered by a change of coordinates into the form (10-12).

Consider nonlinear systems of the form (1). Notice that, in general, observability (resp. rank observability) of system (1) does not imply observability (resp. rank observability) of the associated autonomous system:

$$
\left(\Sigma_{u}\right) \quad\left\{\begin{array}{l}
\dot{x}=f_{u}(x) \\
y=h(x)
\end{array}\right.
$$

where $u$ is a fixed constant control and $f_{u}(x)=f(u, x)$.

The uniform observability structure that we will define in particular possesses the property that if system (1) is rank observable, then for every fixed $u \in U,\left(\Sigma_{u}\right)$ defined by (40) is also rank observable.

To do so, let $u \in U$ and consider the following codistributions:

- $\mathcal{E}_{1}^{u}$ is spanned by $\left\{d h_{1}, \ldots, d h_{p}\right\}$ (notice that $\mathcal{E}_{1}^{u}$ does not depend on $u$ since $h_{i}=h_{i}(x)$ );

- for $k \geq 1$, let $\mathcal{E}_{k+1}^{u}$ be the codistribution spanned by $\mathcal{E}_{k}^{u}$ and $\left\{d L_{f_{u}}^{k}\left(h_{1}\right), \ldots, d L_{f_{u}}^{k}\left(h_{p}\right)\right\}$.

Clearly, we have $\mathcal{E}_{1}^{u} \subset \ldots \subset \mathcal{E}_{n-1}^{u} \subset \mathcal{E}_{n}^{u} \subset \ldots$

Definition 4.1. System (1) is said to have a $U$-uniform observable structure (U-u.o.s) if and only if:

(i) $\forall u, u^{\prime} \in U ; \forall x \in M, \mathcal{E}_{k}^{u}(x)=\mathcal{E}_{k}^{u^{\prime}}(x)$;

(ii) for each $i$, the codistribution $\mathcal{E}_{i}^{u}$ is of constant dimension $\nu_{i}^{u}\left(\operatorname{dim} \mathcal{E}_{i}^{u}(x)=\nu_{i}^{u}, \forall x \in M ; \forall u \in U\right)$.

In a similar way, let $\left(\mathcal{E}_{i}\right)_{i \geq 1}$ be the family of codistributions defined by:

- $\mathcal{E}_{1}=\operatorname{span}\left\{d h_{1}, \ldots, d h_{p}\right\}$

- $\mathcal{E}_{i+1}=\mathcal{E}_{i}+\operatorname{span}\left\{d L_{f_{u_{i}}} \ldots L_{f_{u_{1}}}\left(h_{j}\right) ; u_{1}, \ldots, u_{i} \in U, j=1, \ldots, p\right\}$.

\section{Remark 4.1.}

a) From (i), we can deduce that for every $u \in U$ and every $i \geq 1, \mathcal{E}_{i}=\mathcal{E}_{i}^{u}$.

b) From (ii), if system (1) is rank observable at some $x$ and has a $U$-u.o.s., then it is rank observable at each point of $M$.

According to Remark 4.1, we shall denote indifferently $\mathcal{E}_{k}^{u}$ by $\mathcal{E}_{k}, \nu_{k}^{u}$ by $\nu_{k}$ and we shall denote by $q$ the smallest integer s.t. $\mathcal{E}_{q}=\mathcal{E}_{q+1}$.

In what follows, we assume that $U$ is such that every compact subset of $U$ is also a compact subset of $\mathbb{R}^{m}$. This property holds in particular if $U$ is a closed or an open subset of $\mathbb{R}^{m}$. For the sake of simplicity, we will also assume that $h$ is a local submersion.

We now state the main result of this section:

Theorem 4.1. Assume that system (1) is rank observable at some point of $M$ and has a $U$-u.o.s. Then, for every compact subset $U^{\prime}$ of $U$; system (1) is locally $U^{\prime}$-uniformly observable (see Def. 4.1).

Remark 4.2. Notice that if we omit the compactness hypothesis of $U^{\prime}$, the theorem is no longer true. 
Indeed, consider the following example:

$$
\left\{\begin{array}{l}
\dot{x}_{1}=\cos \left(1+u^{2}\right) x_{3} \\
\dot{x}_{2}=\sin \left(1+u^{2}\right) x_{3} \\
\dot{x}_{3}=0 \\
y=\left(x_{1}, x_{2}\right)
\end{array}\right.
$$

with $U=\mathbb{R}$ and $M=\mathbb{R}^{3}$. Clearly, $\mathcal{E}_{1}^{u}$ and $\mathcal{E}_{2}^{u}$ are respectively spanned by $\left\{d x_{1}, d x_{2}\right\}$ and $\left\{d x_{1}, d x_{2}, d x_{3}\right\}$. Thus (41) is rank observable and it has a $\mathbb{R}$-u.o.s.

Now, taking any $x^{0}=\left(x_{1}^{0}, x_{2}^{0}, x_{3}^{0}\right) \in \mathbb{R}^{3}$ and any neighborhood $\left.V_{x^{0}}^{\varepsilon}=\right] x_{1}^{0}-\varepsilon, x_{1}^{0}+\varepsilon[\times] x_{2}^{0}-\varepsilon, x_{2}^{0}+\varepsilon[\times] x_{3}^{0}-\varepsilon, x_{3}^{0}+$ $\varepsilon$ [. Consider any constant control $u$ such that $\frac{2 k \pi}{1+u^{2}}<\varepsilon$ and taking two initial states $x, \bar{x}$ with $x_{1}=\bar{x}_{1}, x_{2}=\bar{x}_{2}$ and $x_{3}-\bar{x}_{3}=\frac{2 k \pi}{1+u^{2}}$. Let $x(\cdot), \bar{x}(\cdot)$ be the trajectories corresponding to $u$ and respectively issued from $x$ and $\bar{x}$. Obviously, $x_{1}(t)=\bar{x}_{1}(t)$ and $x_{2}(t)=\bar{x}_{2}(t)$ for every $t \geq 0$. Thus, such $u$ is not universal on any $[0, T], T>0$. Hence system (41) restricted to $V_{x^{0}}^{\varepsilon}$ is not locally $U^{\prime}$-uniformly observable for any unbounded interval of $U^{\prime}$ of $\mathbb{R}$.

The proof of Theorem 4.1 requires the following lemma:

Lemma 4.1. Assume that system (1) is rank observable at some point and has a U-u.o.s. Then:

i) for every $x \in M$, there exist a neighbourhood $V$ and a diffeomorphim: $\Phi: V \longrightarrow W$ which transforms system (1) restricted to $V$ into the following form:

$$
\left\{\begin{array}{l}
\dot{z}^{1}=F^{1}\left(u, z^{1}, z^{2}\right) \\
\dot{z}^{2}=F^{2}\left(u, z^{1}, z^{2}, z^{3}\right) \\
\quad \cdots \\
\dot{z}^{q}=F^{q}(u, z) \\
y=z^{1}
\end{array}\right.
$$

where

$$
z^{i} \in \mathbb{R}^{n_{i}}, \quad z=\left[\begin{array}{c}
z^{1} \\
\vdots \\
z^{q}
\end{array}\right] \in W, \quad u \in U
$$

Moreover, we have

$$
p=n_{1} \geq n_{2} \geq \ldots \geq n_{q}
$$

ii) $\forall u \in U ; \forall z \in W ; \forall i, 1 \leq i \leq q-1$, we have:

$$
\operatorname{rank}\left(\frac{\partial F^{i}}{\partial z^{i+1}}(u, z)\right)=n_{i+1}
$$

where $n_{i+1}=\operatorname{dim} \mathcal{E}_{i+1}-\operatorname{dim} \mathcal{E}_{i}$.

Proof of Lemma 4.1. Since system (1) has a $U$-u.o.s., we have: $\forall u \in U, \mathcal{E}_{1}^{u}=\mathcal{E}_{1} \subset \mathcal{E}_{2}^{u}=\mathcal{E}_{2} \subset \ldots \subset \mathcal{E}_{q}^{u}=\mathcal{E}_{q}$ and for $i \geq q+1, \mathcal{E}_{i}^{u}=\mathcal{E}_{i}=\mathcal{E}_{q}^{u}$.

Now, let $u^{\circ}$ be a fixed element of $U$; from the definition of the $\mathcal{E}_{i}^{u^{\circ}}$ 's, we know that $\left(d h_{1}, \ldots, d h_{p}\right)$ forms a basis of $\mathcal{E}_{1}=\mathcal{E}_{1}^{u^{\circ}}$ (since $h: M \longrightarrow \mathbb{R}^{p}$ is assumed to be an almost everywhere local submersion). For 
$i=2, \ldots, q$, and after reordering adequately $\left(h_{1}, \ldots, h_{p}\right)$, a basis of $\mathcal{E}_{i}=\mathcal{E}_{i}^{u^{\circ}}$ is given by

$$
\mathcal{B}_{i}=\left(d h_{1}, \ldots, d h_{p}, d L_{f_{u^{\circ}}}\left(h_{1}\right), \ldots, d L_{f_{u^{\circ}}}\left(h_{n_{2}}\right), \ldots, d L_{f_{u^{\circ}}}^{i-1}\left(h_{1}\right), \ldots, d L_{f_{u^{\circ}}}^{i-1}\left(h_{n_{i}-1}\right)\right)
$$

Set $n_{1}=p$ and $n_{i}=\operatorname{dim} \mathcal{E}_{i}-\operatorname{dim} \mathcal{E}_{i-1}$, and using the construction of the $\mathcal{B}_{i}$, we obtain $n_{1} \geq n_{2} \geq \ldots \geq n_{q}$.

Now, using the fact that system (1) is rank observable at some point, from Remark 4.1, it becomes rank observable at any point of $M$ and hence, the dimension of $\mathcal{E}_{q}=\mathcal{E}_{q}^{u^{\circ}}$ is equal to $n$. Moreover, from Definition 4.1(ii), it follows that $\Phi=\left(h_{1}, \ldots, h_{n_{1}}, \ldots, L_{f_{u^{\circ}}}^{n_{q}-1}\left(h_{1}\right), \ldots, d L_{f_{u^{\circ}}}^{n_{q}-1}\left(h_{n_{q}}\right)\right)$ becomes a local diffeomorphism around each point of $M$. Now, a simple calculation shows that for every $x \in M$; there exists a neighbourhood $V$ of $x$ such that system (1) restricted to $V$ can be transformed by $\Phi$ into a system of the form (42). Indeed, since $\mathcal{E} \hat{u}_{k}=\mathcal{E}_{k}^{u^{\circ}}, \forall u$, we have $L_{f_{u}}\left(L_{f_{u^{\circ}}}^{i-1}\left(h_{j}\right)\right)$ depends only on $h_{1}, \ldots, h_{n_{1}}, \ldots, L_{f_{u^{\circ}}}\left(h_{1}\right), \ldots, L_{f_{u^{\circ}}}^{i}\left(h_{n_{i+1}}\right)$.

To end the proof of the lemma, it remains to prove (44).

Denote by $\widetilde{\mathcal{E}}_{i}, \widetilde{\mathcal{E}}_{i}^{u}$ the codistributions associated to system (42) defined in a similar manner as the $\mathcal{E}_{i}$ 's and $\mathcal{E}_{i}^{u}$ 's. Notice that $\mathcal{E}_{i}\left(\right.$ resp. $\left.\mathcal{E}_{i}^{u}\right)$ is the pull-back of $\widetilde{\mathcal{E}}_{i}\left(\right.$ resp. $\left.\widetilde{\mathcal{E}}_{i}^{u}\right)\left(\mathcal{E}_{i}=\Phi_{\star} \widetilde{\mathcal{E}}_{i}\left(\right.\right.$ resp. $\left.\left.\mathcal{E}_{i}^{u}=\Phi_{\star} \widetilde{\mathcal{E}}_{i}^{u}\right)\right)$. Since $\Phi$ is a diffeomorphism, the properties (i) and (ii) of Definition 4.1 are then preserved for the $\widetilde{\mathcal{E}}_{i}^{u}$ 's. But $\widetilde{\mathcal{E}}_{i}^{u}$ is spanned by $\left(d z_{1}^{1}, \ldots, d z_{n_{1}}^{1}, \ldots, \frac{\partial F_{1}^{i-1}}{\partial z^{i}} d z^{i}, \ldots, \frac{\partial F_{n_{i}-1}^{i-1}}{\partial z^{i}} d z^{i}\right)$, where

$$
\frac{\partial F_{j}^{i-1}}{\partial z^{i}}=\left(\frac{\partial F_{j}^{i-1}}{\partial z_{1}^{i}}, \ldots, \frac{\partial F_{j}^{i-1}}{\partial z_{n_{i}}^{i}}\right) \text { and } d z^{i}=\left(\begin{array}{c}
d z_{1}^{i} \\
\vdots \\
d z_{n_{i}}^{i}
\end{array}\right) .
$$

Since $\widetilde{\mathcal{E}}_{i}^{u}$ is of a constant dimension $\sum_{j=1}^{i} n_{j}$, it follows that:

$$
\operatorname{rank} \frac{\partial F^{i-1}}{\partial z^{i}}(u, z)=n_{i}, \forall(u, z) \in U \times W \text { where } W=\Phi(V) .
$$

We arrive now at the proof of Theorem 4.1.

Proof of Theorem 4.1. Let $U^{\prime}$ be a compact subset of $U$. Let us show that for every $x \in M$; there exists a neighborhood $V_{x}$ of $x$ such that the restriction of system (1) to $V_{x}$ is $U^{\prime}$-uniformly observable. Using Lemma 4.1 and the fact that the observability is an intrinsic property (it does not depend on the system of coordinates), it suffices to show that the restriction of system (42) to $W=\Phi\left(V_{x}\right)$ is $U^{\prime}$ uniformly observable.

To do so, we need the following notations:

Set $\nu_{i}=n_{1}+\ldots+n_{i}$ the dimension of $\mathcal{E}_{i}$, and denote by $\pi_{i}$ (resp. $\underline{\pi}_{i}$ ) the canonical projection from $\mathbb{R}^{\nu_{i}}$ to $\mathbb{R}^{n_{i}}$ defined by $\left(z^{1}, \ldots, z^{i}\right) \mapsto z^{i}$ (resp. from $\mathbb{R}^{\nu_{i}+1}$ to $\mathbb{R}^{\nu_{i}}:\left(z^{1}, \ldots, z^{i+1}\right) \mapsto\left(z^{1}, \ldots, z^{i}\right)$ ).

Set $W_{i}=\pi_{i}(W), \underline{W}_{i}=\underline{\pi}_{i}(W), \underline{z}^{i}=\left(z^{1}, \ldots, z^{i}\right)$ and denote by $F_{u, \underline{z}^{i}}$ the map from $W_{i+1}$ into $\mathbb{R}^{n_{i}}$ defined by $F_{u, \underline{z}^{i}}\left(z^{i+1}\right)=F^{i}\left(u, \underline{z}^{i}, z^{i+1}\right)$, where the $F^{i}$ 's are defined in (42).

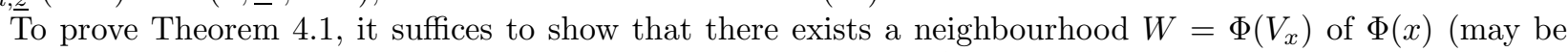
small) such that for $1 \leq i \leq q-1$; for every $u \in U^{\prime}$ and for every $\underline{z}^{i} \in \underline{W}_{i}, F_{u, \underline{z}^{i}}^{i}$ is one to one. Indeed, assume that the $F_{u, \underline{z}^{i}}^{i}$ 's are one to one and let us show that system (42) restricted to $\underline{W}$ is $U^{\prime}$-uniformly observable.

Let $u^{\circ}(\cdot) \in L^{\infty}\left([0, T], U^{\prime}\right)$ be any admissible input, we will show that $u^{\circ}(\cdot)$ is an universal input on $[0, T]$. Otherwise said, let $z, \bar{z}$ be two initial states such that the corresponding output $z^{1}(t), \bar{z}^{1}(t)$ are identically equal on $[0, T]$ and let us show that $z=\bar{z}$. 
Since $z^{1}(t)=\bar{z}^{1}(t), \forall t \in[0, T]$, differentiating this equality, we get:

$$
\begin{aligned}
F^{1}\left(u^{\circ}(t), z^{1}(t), z^{2}(t)\right) & =F^{1}\left(u^{\circ}(t), \bar{z}^{1}(t), \bar{z}^{2}(t)\right) \\
& =F^{1}\left(u^{\circ}(t), z^{1}(t), \bar{z}^{2}(t)\right)
\end{aligned}
$$

thus,

$$
F_{u^{\circ}(t), z^{1}(t)}^{1}\left(z^{2}(t)\right)=F_{u^{\circ}(t), \bar{z}^{1}(t)}^{1}\left(\bar{z}^{2}(t)\right)
$$

hence,

$$
z^{2}(t)=\bar{z}^{2}(t) \quad \text { (since } F_{u, z^{1}}^{1} \text { is one to one). }
$$

Differentiating this equality and proceeding in a similar way, we get $z^{3}(t)=\bar{z}^{3}(t)$. Repeating this procedure and using the same arguments for $i=3, \ldots$, we get $z=\bar{z}$.

Now, let us show the injectivity of the $F_{u, z^{i}}^{i}$ 's. Assume that for every neighbourhood $W$ of $\Phi(x)$, there exist $i, 1 \leq i \leq q-1 ; u \in U^{\prime}$ and $\underline{z}^{i} \in \underline{W}_{i}$ such that the restriction of $F_{u, \underline{z}^{i}}^{i}$ is not injective. Thus, one can find sequences $\left(\varepsilon_{k}\right)_{k \geq 0}\left(\varepsilon_{k}>0, \lim _{k \rightarrow+\infty} \varepsilon_{k}=0\right),\left(u_{k}\right)_{k \geq 0}, u_{k} \in U^{\prime}$ and $\left(z_{\varepsilon_{k}}\right)_{k \geq 0} \in B\left(\Phi(x), \varepsilon_{k}\right)=\left\{z /\|z-\Phi(x)\|<\varepsilon_{k}\right\}$ such that $F_{u_{k}, \underline{z}_{\varepsilon_{k}}^{i_{0}}}^{i_{0}}$ is not one to one for some fixed $i_{0} \in\{1, \ldots, q-1\}$. It means that, $\forall k ; \exists z_{\varepsilon_{k}}^{i_{0}+1}, \bar{z}_{\varepsilon_{k}}^{i_{0}+1} \in$ $\pi_{k}\left(B\left(\Phi(x), \varepsilon_{k}\right)\right), z_{\varepsilon_{k}}^{i_{0}+1} \neq \bar{z}_{\varepsilon_{k}}^{i_{0}+1}$ and such that $F_{u_{k}, \underline{z}_{\varepsilon_{k}}}^{i_{0}}\left(z_{\varepsilon_{k}}^{i_{0}+1}\right)=F_{u_{k}, \underline{z}_{\varepsilon_{k}}}^{i_{0}}\left(\bar{z}_{\varepsilon_{k}}^{i_{0}+1}\right)$.

Applying the Mean Value theorem, we get:

$$
\left[\frac{\partial F^{i_{0}}}{\partial z^{i+1}}\left(z_{\varepsilon_{k+1}}^{i_{0}+1}+\Theta_{i_{0}}\left(z_{\varepsilon_{k}}^{i_{0}+1}-\bar{z}_{\varepsilon_{k}}^{i_{0}+1}\right)\right)\right] \cdot\left(z_{\varepsilon_{k}}^{i_{0}+1}-\bar{z}_{\varepsilon_{k}}^{i_{0}+1}\right)=0
$$

where $\Theta_{i_{0}}$ is the $n_{i_{0}+1} \times n_{i_{0}+1}$ diagonal matrix $\operatorname{diag}\left(\theta_{1}, \ldots, \theta_{n_{i_{0}+1}}\right)$ for some $\theta_{j} \in[0,1], 1 \leq j \leq n_{i_{0}+1}$.

Since $z_{\varepsilon_{k}}^{i_{0}+1} \neq \bar{z}_{\varepsilon_{k}}^{i_{0}+1}$, set $\zeta_{\varepsilon_{k}}^{i_{0}+1}=\frac{z_{\varepsilon_{k}}^{i_{0}+1}-\bar{z}_{\varepsilon_{k}}^{i_{0}+1}}{\left\|z_{\varepsilon_{k}}^{i_{0}+1}-\bar{z}_{\varepsilon_{k}}^{i_{0}+1}\right\|}$, we obtain:

$$
\left[\frac{\partial F^{i_{0}}}{\partial z^{i+1}}\left(z_{\varepsilon_{k+1}}^{i_{0}+1}+\Theta_{i}\left(z_{\varepsilon_{k+1}}^{i_{0}+1}-\bar{z}_{\varepsilon_{k+1}}^{i_{0}+1}\right)\right)\right] \cdot \zeta_{\varepsilon_{k}}^{i_{0}+1}=0 \text { and }\left\|\zeta_{\varepsilon_{k}}^{i_{0}+1}\right\|=1
$$

Since $\left(u_{k}\right)_{k \geq 1},\left(\zeta_{\varepsilon_{k}}^{i_{0}+1}\right)_{k \geq 1}$ are bounded sequences, then one can extract subsequences $\left(u_{k_{l}}\right)_{l \geq 1},\left(\zeta_{\varepsilon_{k_{l}}}^{i_{0}+1}\right)_{l \geq 1}$ such that $\lim _{k_{l} \rightarrow+\infty} u_{k_{l}}=u$ and $\lim _{k_{l} \rightarrow+\infty} \zeta_{\varepsilon_{k_{l}}}^{i_{0}+1}=\zeta^{i_{0}+1}$ with $u \in U^{\prime}$ and $\left\|\zeta^{i_{0}+1}\right\|=1$.

Now, using the continuity of the map: $\left(u, z^{1}, \ldots, z^{i_{0}+1}\right) \rightarrow \frac{\partial F^{i_{0}}}{\partial z^{i+1}}\left(u, z^{1}, \ldots, z^{i_{0}+1}\right)$ and the fact that $\lim _{k \rightarrow+\infty}\left(z_{\varepsilon_{k_{l}}}^{i_{0}+1}+\Theta_{i_{0}}\left(z_{\varepsilon_{k_{l}}}^{i_{0}+1}-\bar{z}_{\varepsilon_{k_{l}}}^{i_{0}+1}\right)\right)=z^{i_{0}+1}$ and $\lim _{k \rightarrow+\infty} \underline{\varepsilon}_{\varepsilon_{k_{l}}}^{i_{0}}=\underline{z}^{i_{0}}$ we obtain:

$$
\frac{\partial F^{i_{0}}}{\partial z^{i+1}}\left(u, z^{1}, \ldots, z^{i_{0}+1}\right) \zeta^{i_{0}+1}=0 \quad \text { with } \quad\left\|\zeta^{i_{0}+1}\right\|=1 .
$$

But, from Lemma 4.1, the $n_{i_{0}} \times n_{i_{0}+1}$ matrix $\frac{\partial F^{i_{0}}}{\partial z^{i+1}}(u, z)$ is of rank $n_{i_{0}+1}$ and $n_{i_{0}} \geq n_{i_{0}+1}$, thus ker $\frac{\partial F^{i_{0}}}{\partial z^{i+1}}(u, z)=$ $\{0\}$. This is in contradiction with (45).

Conclusion. In this paper, a canonical form for uniformly observable multi-output system is first presented. Then, two observers have been synthesized on the basis of this form. The first has a constant gain whereas the second assumes a gain which depends on the inputs. Finally, we have discussed the characterization of the class of nonlinear systems that can be steered by a change of coordinates to the considered canonical form. 


\section{REFERENCES}

[1] G. Besançon and H. Hammouri, On uniform observation of non uniformly observable systems. Systems Control Lett. 29 (1996) 9-19.

[2] G. Besançon and H. Hammouri, On observer design for interconnected systems. J. Math. Systems Estim. Control 8 (1998).

[3] G. Bornard and H. Hammouri, A high gain observer for a class of uniformly observable systems, in Proc. 30th IEEE Conference on Decision and Control Brighton 122 (1991) 176-192.

[4] J.P. Gauthier and G. Bornard, Observability for any u(t) of a class of nonlinear systems. IEEE Trans. Automat. Control 26 (1981) 922-926.

[5] J.P. Gauthier, H. Hammouri and S. Othman, A simple observer for nonlinear systems - Application to bioreactors. IEEE Trans. Automat. Control 37 (1992) 875-880.

[6] J.P. Gauthier and I.A.K. Kupka, Observability and observers for nonlinear systems. SIAM J. Control Optim. 32 (1994) 975-994.

[7] J.P. Gauthier and I.A.K. Kupka, Observability for systems with more outputs than inputs. Math. Z. 223 (1996) 47-78.

[8] J.P. Gauthier and I.A.K. Kupka, Deterministic Observation Theory and Applications. Cambridge University Press (2001).

[9] R. Hermann and A.J. Krener, Nonlinear controllability and observability. IEEE Trans. Automat. Control 22 (1977) $728-740$.

[10] A. Isidori, Nonlinear control systems: An introducion, Vol. 72. Springer, Berlin (1985).

[11] A.J. Krener and A. Isidori, Linearization by output injection and nonlinear observers. System Control Lett. 3 (1983) $47-52$.

[12] A.J. Krener and W. Respondek, Nonlinear observers with linealizable error dynamics. SIAM J. Control Optim. 23 (1985) 197-216.

[13] H.J. Sussman, Single-input observability of continuous-time systems. Math. System Theory 12 (1979) 371-393.

[14] F.E. Thau, Observing the state of nonlinear dynamics systems. Int. J. Control 17 (1973) 471-479.

[15] D. Williamson, Observability of bilinear systems, with application to biological control. Automatica 32 (1977) $143-254$. 